S80 A CLINICAL EVALUATION OF QUANTITATIVE, CULTUREINDEPENDENT METHODS FOR THE IDENTIFICATION OF BACTERIA IN CYSTIC FIBROSIS SPUTUM AND BRONCHOALVEOLAR LAVAGE FLUIDS

doi:10.1136/thoraxjnl-2012-202678.086

${ }^{1} \mathrm{C}$ Holmes, ${ }^{2} \mathrm{~J}$ Shah, ${ }^{\mathrm{D}} \mathrm{D}$ Modha, ${ }^{2} \mathrm{R}$ Herzallah, ${ }^{1} \mathrm{H}$ Patel, ${ }^{2} \mathrm{~K}$ Haldar, ${ }^{2} \mathrm{M}$ Barer, ${ }^{2 \mathrm{~K}}$ Rajakumar, ${ }^{2}$ E Gaillard. 'University Hospitals of Leicester NHS Trust, Leicester, UK; ' ${ }^{2}$ niversity of Leicester, Leicester, UK

Introduction and objectives: The detection of bacteria contributing to respiratory exacerbations in cystic fibrosis patients is routinely performed using standardised microbiological culture techniques. These methods often fail to identify a significant pathogen in symptomatic patients and have been found to underestimate the burden of fastidious organisms such as Streptococcus pneumoniae in CF sputum. There is also increasing evidence linking respiratory exacerbations to infection with organisms that are not identified by routine microbiology.

The primary objective of this cross-sectional study was to test the agreement between standard microbiology and molecular (culture-independent) techniques in detecting five common pathogens in sputum of children with CF.

Methods Culture-independent microbiology was performed on samples obtained from children experiencing respiratory exacerbations over a 12 month period from April 2010 to March 2011. Sputum samples were either produced spontaneously or obtained either by sputum induction with hyper tonic saline or bronchoalveolar lavage. Aliquots of sputum we refrozen either neat or mixed 1:1 with $0.1 \%$ dithiothreiotol, prior to batch extraction of total nucleic acid. Total bacterial load was determined using abroad-range $16 \mathrm{~S}$ rDNA quantitative real-time PCR. Specific real-time qPCR assays were used to quantify the numbers of Staphylococcus aureus, Haemophilus influenzae, S pneumoniae, Pseudomonas aeruginosa and Moraxella catarrhalis. Routine microbiology was performed by adhering to the laboratory standards published by the UK CF Trust.

Results Fifty five samples (44 sputum and 11 bronchoalveolar lavage fluid) from 33 children were available for molecular analysis. In 40 , the total bacterial load was calculated to be $10^{7}$ or greater per $\mathrm{ml}$ of fluid. The qPCRs detected clinically relevant pathogens at significant levels $(>1 \%$ of total) more frequently than standard microbiology.

Conclusion We found that standard microbiology alone revealed fewer of the clinically relevant respiratory pathogens studied compared to molecular techniques. PCR based analysis also offers the potential to identify pathogens more rapidly and to detect organisms that are difficult to detect by routine culture. This may have an impact on the choice of antibiotic for the treatment of an exacerbation. Compared to standard microbiology, however, these assays are expensive and not readily available for routine diagnostics.

\section{S81 LUNG BIOPSY FOR CHRONIC PULMONARY DISEASE IN CHILDREN}

doi:10.1136/thoraxjnl-2012-202678.087

${ }^{1} \mathrm{RM}$ Greenhalgh, ${ }^{2} \mathrm{Mr}$ IE Yardley, ${ }^{3} \mathrm{Dr} \mathrm{F}$ Child, ${ }^{4} \mathrm{Mr} \mathrm{J}$ Bruce, ${ }^{4}$ Miss GME Humphrey ${ }^{1}$ Final Year Medical Student, The University of Nottingham, Nottingham, UK; '2Specialist Registrar in Paediatric Surgery, Royal Manchester Children's Hospital, Oxford Road, Manchester M13 9WL, UK; ${ }^{3}$ Consultant in Paediatric Respiratory Medicine, Royal Manchester Children's Hospital, Oxford Road, Manchester M13 9WL, UK; ${ }^{4}$ Consultant Paediatric Surgeon, Royal Manchester Children's Hospital, Oxford Road, Manchester M13 9WL, UK

Introduction and Objectives Lung biopsy is frequently used in the management of children with chronic pulmonary disease to obtain a histological diagnosis. Previous studies have reported a high diagnostic yield and a low complication rate following lung biopsy in children. We set out to further evaluate the role of lung biopsy in this group of clinically challenging patients by reviewing our experience of this procedure.

Methods A retrospective case-note review was carried out of all patients under 18 yrs who underwent a lung biopsy from 1998 to 2011. Data collected included age, mode of biopsy, histological findings, pre and post biopsy diagnosis, pre and post biopsy treatment, and complications of the procedure.

Results Thirty three children (12 boys) underwent lung biopsy in the period studied. Their ages ranged from three months to 16 years (median 5 years 5 months). Five biopsies were obtained using a thoracoscopic approach, the remainder via thoracotomy. Following the procedure, 17 patients required ventilation on the intensive care unit for a median of two days. Eight children (24\%) experienced a direct complication of the procedure. Seven required an intercostal drain for a pneumothorax, one a tension pneumothorax presenting six days post-operatively. The operative mortality was $12 \%(4 / 33)$. Three children (9\%) died within 28 days of surgery, two due to progressive respiratory failure and one following pneumonia. One patient died three months post-operatively due to a persistent air leak from the biopsy site. Twenty-six (79\%) biopsies provided a sample adequate for definitive histological diagnosis. In $16(48 \%)$ children the working diagnosis and treatment was changed following lung biopsy.

Conclusion Lung biopsy retains a place in the management of children with chronic pulmonary disease when there is diagnostic doubt. However it carries a significant morbidity and mortality which must be borne in mind when considering the need for histological diagnosis.

\section{S82 AIRWAY INFLAMMATION IS PRESENT BY 4 MONTHS IN CF INFANTS DIAGNOSED ON NEWBORN SCREENING}

doi:10.1136/thoraxjnl-2012-202678.088

'RM Thursfield, ${ }^{2} \mathrm{~A}$ Bush, 'EWFW Alton, ${ }^{1} \mathrm{JC}$ Davies. 'National Heart and Lung Institute, Imperial College, London, UK; ${ }^{2}$ Department of respiratory paediatrics, Royal Brompton Hospital, London, UK

Introduction Cystic fibrosis (CF) newborn screening (NBS) allows early introduction of treatment, often before any symptoms arise with the aim of reducing airway infection and inflammation. UK nationwide screening began in 2007.

Aims It is usual clinical practise at our centre to perform fibreoptic bronchoscopy (FOB) at the age of 3 months for all children with CF. The aim of this study was to establish the presence of infection and degree of inflammation in airways in the NBS cohort. Bronchoalveolar lavage fluid (BALF) was cultured and examined for cellular inflammation.

Results Infants diagnosed by NBS undergoing routine FOB who had either BALF absolute cell count or differential cell count assessed were included in the study. 44 infants (48\% female), median age (range) 15 (7-28) weeks met these criteria. The majority of these infants were symptom free however 13 (29\%) had bacterial isolates from their BALF. Comparable data are also available for 71 children with established CF (median age 9.5 years; range 1.9-16.7 years) of whom 46 (65\%) were BALF culture positive and for 6 healthy controls (median age 12.3 years, range 10.5-15.4 years).

Cellular inflammation was present in the airways in infants diagnosed by NBS, both in those who were culture positive and negative in their BALF. Absolute BALF cell count and neutrophil differential were significantly higher in both NBS and established CF patients compared with healthy controls $(p<0.02)$. Median absolute cell counts and neutrophil differentials can be seen in table 1. For both NBS and established CF, in those who had bacteria isolated from their BALF, an increase in neutrophil differential was seen compared with those culture negative at the time of $\mathrm{FOB}$ although 